\title{
Application of Grey Relational Analysis in Emergency Decision System
}

\author{
Yong Wang ${ }^{1, a}$, Jun Wang ${ }^{2, b}$ \\ ${ }^{1}$ School of Computer Science, Guangdong University of Technology, Guangzhou 510000, China; \\ ${ }^{2}$ School of Computer Science, Guangdong University of Technology, Guangzhou 510000, China. \\ a986715930@qq.com, b2412550795@qq.com
}

Keywords: gray correlation analysis method, emergency decision system, effect evaluation vector, grey incidence matrix.

\begin{abstract}
The gray of the emergency command system includes randomness and fuzziness, as well as other kinds of certainty, uncertainty and incomplete. This paper aims to solve the optimal emergency plan and using the gray correlation analysis method to analyze the data of the emergency index of the emergency decision system. First, through the definition of the gray correlation analysis method to calculate the best effect evaluation vector of each emergency response sequence. Then the system grey incidence matrix is established to calculate the weight value of each index. Finally, the effect grade of grey correlation degree and emergency plan is obtained by calculating and determining the best emergency plan, which is more objective and reasonable to exert the decision-making function of the emergency decision system.
\end{abstract}

\section{Introduction}

At present, there are mainly fuzzy evaluation method, catastrophe theory analysis method, analytic hierarchy process, computer simulation evaluation method and so on to solve the decision scheme of the emergency command system. Wang Zhen[1] apply interval analytic hierarchy process (IAHP) for the construction of emergency command system of fire safety factors which were quantitative and qualitative assessment, However, the method is used to deal with the data in the emergency command system with the traditional mathematical method, which is not necessarily appropriate for the application of the emergency decision system. Li Gai[2] numerical simulation analysis of the data of the emergency command system, but the fire emergency command system can not be analyzed from the whole macro point of view.

Grey incidence analysis method (Relational Analysis Grey) is a kind of grey system analysis method, which is an effective method to measure the degree of correlation among all kinds of decision schemes. The purpose of the analysis is to determine the close degree of each decision-making method by comparing the curve shape of the data sequence. Under normal circumstances, the closer the geometry, the more close to the trend of change and the greater the degree of correlation. In short, the gray correlation analysis method is to calculate the grey correlation degree of the data sequence and the system characteristic data sequence by calculating the correlation factor variable. To obtain the best solution, the analysis of the optimal solution can be made to help the decision maker to make decision analysis. The grey incidence analysis method is used to analyze the data of the emergency command system[3], and the decision method can be effectively used to make up the deficiency of other methods and it can effectively make up for the shortcomings of other methods to determine, which makes the emergency decision system more objective and reasonable.

\section{Introduction of related technologies}

\subsection{Basic concepts of grey decision.}

Definition 1. All the events within the scope of the study are called event sets. Given a set $X=\left\{x_{1}, x_{2}, x_{3}, \ldots ., x_{n}\right\}, x_{i}(i=1,2,3, \ldots, n)$ is the $\mathrm{i}$-th event,Given a set $Y=\left\{y_{1}, y_{2}, \ldots, y_{n}\right\}$, and $y_{j}(j=1,2,3, \ldots, n)$ is the $\mathrm{j}$-th strategy. 
Definition 2. Event set $X=\left\{x_{1}, x_{2}, \ldots, x_{n}\right\}$ and strategy set $Y=\left\{y_{1}, y_{2}, y_{3}, \ldots, y_{n}\right\}$ cartesian product $X \otimes Y=\left\{\left(x_{i}, y_{j}\right) \mid x_{i} \in X, y_{j} \in Y\right\}$ is called situation set, recorded as $S=X \otimes Y$.for arbitrary $x_{i} \in X, Y_{j} \in Y,\left(x_{i}, y_{j}\right)$ is called situation, recorded as $s_{i j}=\left(x_{i}, y_{j}\right)$.

Definition 3. Suppose $t_{i_{0} j_{0}}=\left[t_{i_{0} j_{0}}{ }^{(1)}, \ldots, t_{i_{0} j_{0}}{ }^{(s)}\right]$ is the optimal decision vector. $S=\left\{s_{i j}\right\}$ is the situation set. if $t_{i_{0} j_{0}} \notin S$, then $t_{i_{0} j_{0}}$ is the ideal scheme effect evaluation vector.

Definition 4. Suppose $S=\left\{s_{i j}\right\}$ is the situation set. Situation $s_{i j}$ corresponding effect vector $t_{i j}, i=1,2, \ldots, n, j=(1,2, \ldots, n)$, which is called the optimal ideal vector. Grey absolute correlation degree between $t_{i j}$ and $t_{i_{0} j_{0}}$ is $\xi_{i j}$, if for arbitrary $i$ and $i \neq i_{1}$, for arbitrary $\mathrm{j}$ and $j \neq j_{1}$, always have $\xi_{i_{1} j_{1}} \geq \xi_{i j}, t_{i_{1} j_{1}}$ is the sub optimal effect vector, and $s_{i_{1} j_{1}}$ is the sub optimal situation.

\subsection{Calculating gray correlation coefficient.}

The correlation degree of each factor is based on the similarity degree of the curve shape. The difference of the curve can be used as the criterion of the decision scheme. Supposing $T=\left\{t_{i} \mid i \in\{1,2, \ldots, n\}, n \geq 2, t_{i}(k) \in t_{i}\right\}, t_{0}(k)$ and $t_{i}(k)$ is $t_{0}$ and $t_{i}$ at the k-th point data. if $\xi_{0 i}(k)$ is $T$ 's comparison between $t_{0}(k)$ and $t_{i}(k)$ under a certain condition . The smaller the $\left|t_{0}(k)-t_{i}(k)\right|$, the greater the $\xi_{0 i}(k)$. Supposing $\xi_{0 i}(k)$ is the grey correlation coefficient $t_{i}$ to $t_{0}$ at point $\mathrm{k}$.

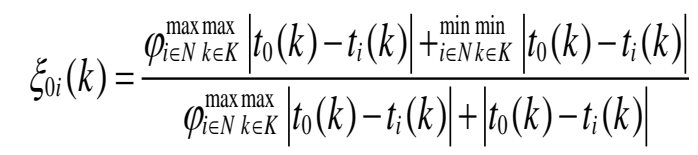

1) $\varphi_{i \in N}^{\max \max }\left|t_{0}(k)-t_{i}(k)\right|$ and $\min _{i \in N k \in K}\left|t_{0}(k)-t_{i}(k)\right|$ is the comparative environment between $t_{i}$ and $t_{0},\left|t_{0}(k)-t_{i}(k)\right|$ is the distance.

2) Constant $\varphi$ is the resolution factor, It can be used to regulate the comparison of the environment, $\varphi \in[0,1]$. When $\varphi$ approaches 1 , the environment will be maintained wholly intact. When $\varphi$ approaches 0 , the environment will disappear. When the value of $\varphi$ is 0.52103 , which is easy to see the law of the degree of correlation. So in general, $\varphi=0.5$.

\subsection{Calculating grey relational degree.}

The magnitude of the association between the two different schemes is called the degree of correlation[4]. The main description of the various indicators of the change of the speed, size and direction of the relative. In other words, the system is in the process of development of the various indicators of the relative changes in the situation. If in the process of development the changes of both remain relatively consistent, it can be considered that the relationship between the two is big. The essence of association analysis is to compare the geometric relations of curves. If the two curves coincide, the correlation is good. However, the two column geometric curve cannot be vertical, which is no correlation, so the correlation coefficient must be greater than zero. Because the correlation coefficient is a measure of the degree of relevance, in the course of the comparison, the correlation coefficient has many, so the average value of all the correlation coefficient is used as the measure of the degree of association in the process of comparison. Given real number $r\left(x_{0}, x_{i}\right)$ is the average value of $\xi_{0 i}(k)$. And,

$$
r\left(x_{0}, x_{i}\right)=\frac{1}{n} \sum_{k=1}^{n} \xi_{0 i}(k)
$$

Which is recorded as the grey relational degree of $x_{i}$ to $x_{0}$. But using this formula to calculate the correlation degree is an important index which takes all the weight of the indicators as equal, and the weight of each index is different in the practical application of the emergency decision system. Therefore, according to the actual situation of the gray correlation coefficient to do the average weighted sum of gray correlation degree. 
According to the difference between the weight of each index, to give the corresponding weight according to the importance $\sigma(k)$, and $\sum_{k=1}^{n} \sigma(k)=1,0 \leq \sigma(k) \leq 1$, According to Shannon's information theory, If the value of random variables $\mathrm{x}$ is $x_{i}$, and the probability value of $x_{i}$ is $p_{i}\left(p_{i}>0\right)$, and in the case of $\sum_{i=1}^{n} p_{i}=1$,Entropy definition of random variable $\mathrm{x}$ is:

$$
F(x)=-c \sum_{i=1}^{n} \log p\left(p_{x i}\right) p\left(x_{i}\right)
$$

And supposing $0 \log 0=0$ Therefore, using the abovetheory, the weight of grey correlation coefficient is expressed as:

$$
\sigma(k)=\frac{1-\lambda(k)}{\sum_{k=1}^{n}(1-\lambda(k)}
$$

Finally, the final weighted correlation degree is:

$$
r\left(x_{0}, x_{i}\right)=\sum_{k=1}^{n} \xi_{0 i} \sigma(k)
$$

\section{Example application and analysis}

In view of the decision function of the gray correlation analysis method in the emergency decision system, this paper uses concrete data to make concrete analysis. According to Emergency Command Management System of a prefecture level city, in order to respond to the best emergency decision, using this program to assist decision makers to make decisions.

When there is a sudden fire in a location, the existing 5 decision alternatives peculiarities, respectively using (R1, R2, R3, R4, R5) to express, and 6 indicators need to be considered. Different indicators have different certainty and uncertainty. Uncertainty index includes fuel element $\left(\mathrm{S}_{1}\right)$, elements of fire spreading $\left(S_{2}\right)$ and factors of fire $\operatorname{scope}\left(S_{3}\right)$. Certainty index includes emergency command level $\left(\mathrm{S}_{4}\right)$, emergency resource situation $\left(\mathrm{S}_{5}\right)$ and case of fire $\left(\mathrm{S}_{6}\right)$. Details are shown in Table 1:

Table 1 Fire Emerency Response Index System

\begin{tabular}{|l|l|l|}
\hline $\begin{array}{l}\text { Target } \\
\text { layer }\end{array}$ & $\begin{array}{l}\text { Criterion } \\
\text { layer }\end{array}$ & Index layer \\
\hline \multirow{4}{*}{$\begin{array}{l}\text { Fire } \\
\text { emergency } \\
\text { response } \\
\text { decision }\end{array}$} & $\begin{array}{l}\text { Uncertainty } \\
\text { factors }\end{array}$ & fuel element(S1) \\
\cline { 2 - 3 } & $\begin{array}{l}\text { Certainty } \\
\text { factors }\end{array}$ & elements of fire spreading(S2) \\
\cline { 3 - 3 } & $\begin{array}{l}\text { factors of fire scope(S3) } \\
\text { emergency command level(S4) } \\
\text { situation(S5) }\end{array}$ \\
\cline { 3 - 3 } & case of fire(S6) \\
\hline
\end{tabular}

The quantitative evaluation values of each index are shown in Table 2:

Table 2 Quantitative Evaluation of Indecators

\begin{tabular}{lcccccc}
\hline & S1 & S2 & S3 & S4 & S5 & S6 \\
\hline R1 & {$[2,5]$} & {$[6,8]$} & {$[9,11]$} & {$[8,17]$} & {$[1,10]$} & {$[10,33]$} \\
R2 & {$[6,8]$} & {$[5,9]$} & {$[8,11]$} & {$[6,21]$} & {$[5,7]$} & {$[80,99]$} \\
R3 & {$[7,9]$} & {$[3,5]$} & {$[6,10]$} & {$[3,17]$} & {$[9,20]$} & {$[34,50]$} \\
R4 & {$[7,9]$} & {$[4,7]$} & {$[7,15]$} & {$[4,16]$} & {$[5,15]$} & {$[43,58]$} \\
R5 & {$[4,6]$} & {$[5,7]$} & {$[8,12]$} & {$[3,19]$} & {$[8,14]$} & {$[35,40]$} \\
\hline
\end{tabular}

Step 1: Determining the best effect evaluation vector .Using the data in Table 2, according to the definition of grey correlation degree, Calculating the best effect evaluation vector:

$$
t_{i 0 j 0}=([0.6812,1],[0.7231,1],[0.8362,1],[0.6759,1],[0.7823,1])
$$


Step 2: Calculating correlation coefficient. According to the best effect evaluation vector and formula (1), the calculation of the correlation coefficient matrix is:

$$
\left[\begin{array}{llllll}
\xi_{1}(1) & \xi_{1}(2) & \xi_{1}(3) & \xi_{1}(4) & \xi_{1}(5) & \xi_{1}(6) \\
\xi_{2}(1) & \xi_{2}(2) & \xi_{2}(3) & \xi_{2}(4) & \xi_{2}(5) & \xi_{2}(6) \\
\xi_{3}(1) & \xi_{3}(2) & \xi_{3}(3) & \xi_{3}(4) & \xi_{3}(5) & \xi_{3}(6) \\
\xi_{4}(1) & \xi_{4}(2) & \xi_{4}(3) & \xi_{4}(4) & \xi_{4}(5) & \xi_{4}(6) \\
\xi_{5}(1) & \xi_{5}(2) & \xi_{5}(3) & \xi_{5}(4) & \xi_{5}(5) & \xi_{5}(6)
\end{array}\right]=\left[\begin{array}{llllll}
0.2842 & 0.9683 & 0.7654 & 0.9661 & 0.8873 & 0.5488 \\
0.9353 & 0.8625 & 0.8532 & 0.9192 & 0.4433 & 0.3625 \\
0.8542 & 0.6814 & 0.5945 & 0.3632 & 0.7273 & 0.8283 \\
0.6895 & 0.9212 & 0.9242 & 0.7552 & 0.3688 & 0.7383 \\
0.9716 & 0.4754 & 0.9639 & 0.9453 & 0.6163 & 0.5244
\end{array}\right]
$$

Step 3: Determing the weight of each index. According to correlation coefficient and formula (3), (4), the weight of each index is: $\mathrm{W}\left(\mathrm{S}_{1}\right)=0.3245, \mathrm{~W}\left(\mathrm{~S}_{2}\right)=0.2746, \mathrm{~W}\left(\mathrm{~S}_{3}\right)=0.3245, \mathrm{~W}\left(\mathrm{~S}_{4}\right)=0.3562$, $\mathrm{W}\left(\mathrm{S}_{5}\right)=0.3749$.

Step 4: Calculation of correlation degree and the determination of emergency plan. According to the weight of each index, the total correlation degree can be obtained through calculation. As follows:

$\mathrm{R}=(\mathrm{R} 1, \mathrm{R} 2, \mathrm{R} 3, \mathrm{R} 4, \mathrm{R} 5)=(0.8254,0.7862,0.6584,0.7249,0.6721)$

According to the size of the correlation degree ordering:R1 > R2 > R4 > R5 > R3.

The value of the grey incidence degree reflects the similarity degree between the emergency plan and the ideal scheme. The greater the value of the gray correlation degree, the higher the degree of similarity between the contingency plan and the ideal scheme. From the point of view of the calculation results, the largest correlation is R1. So it can be concluded that the best emergency decision scheme is R1.

\section{Summary}

In this paper, the grey correlation analysis method is used in the emergency decision system,which solves the emergency decision-making system in the process of determining the emergency indicators and uncertain information. The grey correlation analysis method is simple and feasible, and the analysis result is reliable and intuitive, which makes up for the deficiency of the traditional algorithm for the data analysis of the emergency command system. When the data is analyzed by the gray correlation analysis method, the effective data of all the emergency indicators have an impact on the final decision results, and there is no data loss in the process of analysis. This algorithm can be used in emergency decision system to point out the ideal emergency decision plan, which can help decision makers to make decision.

\section{References}

[1]. Z.Wang, M. liu. “On fire-safety of high-rises with IAHP-based method”. Journal of Safety and Environment.Vol. 6 (2006) No. 12, p. 12-15

[2]. G Li, T Yu, P Lu. "Piston Effect of Elevators in High-rise Building and Analysis of Smoke Control”, China Safety Science Journal.Vol. 19 (2009) No. 2, p. 96-101

[3]. Prze G. "Distances between intuitionistic fuzzy sets and interval-valued fuzzy sets based on the Hausdorff metric”, Fuzzy Sets and System. Vol 148 (2004) No. 23, p.319-323

[4]. C.Chiris. "Implication in intuitionistic fuzzy and interval valued fuzzy set theory:construction,classification,application”, International Journal of Approximate Reasoning. Vol. 35 (2004) No. 14, p 55-59 